\title{
Estilos de liderazgo con tendencia al acoso laboral (Mobbing)
}

\author{
Leadership styles with tendency to the workplace harassment (mobbing)
}

\author{
Alejandra López Tellez, Gloria Lucía López Velásquez, María Esperanza López Duque \\ Facultad de Ingeniería Industrial, Universidad Tecnológica de Pereira, Pereira, Colombia \\ soridutp.edu. co
}

\begin{abstract}
Resumen-El articulo propone, identificar la percepción de acoso en los equipos de trabajo de las organizaciones de diferentes sectores productivos y sus estilos de liderazgo predominantes; a través de un ejercicio investigativo que infiere una serie de variables asociadas a las percepciones de comportamientos acosadores; dicha inferencia se desarrolla a partir de los referentes teóricos y conceptuales abordados en la revisión de literatura internacional respondiendo al contexto legal existente en Colombia. El objeto de investigación se estableció con la participación de 22 grupos de trabajo correspondientes a diferentes niveles jerárquicos de 6 empresas representativas de la región.
\end{abstract}

Palabras clave- Acoso laboral, clima organizacional, estructura organizacional, estilos de liderazgo, percepción, riesgo psicosocial.

Abstract - The article aims to identify the perception of harassment in the work teams of the different productive sectors and their predominant styles of leadership organizations; through a research exercise that infers a series of variables associated with perceptions of harassing behaviors; Such inference is developed from theoretical and conceptual references addressed in the review of international literature in response to the legal context existing in Colombia. The object of investigation was established with the participation of 22 working groups corresponding to different hierarchical levels of 6 representative companies of the region.

Key Word - Harassment at work, organizational climate, organizational structure, styles of leadership, perception, psychosocial risk

\section{INTRODUCCIÓN}

Los estudios sobre las agresiones en el ámbito laboral no son nuevos, el interés que se despertó en los años ' 90 donde la preocupación por la salud de los trabajadores, impulso una revisión a nivel mundial en la prevención de los riesgos laborales. La literatura revela que las mujeres, los jóvenes o los empleados inexpertos, son acosados con más frecuencia y se da en manifiesto en aquellos ambientes laborales de supremo control donde la competitividad y la eficacia a cualquier precio, son respuestas a climas donde priman la ambigüedad, el conflicto de rol y la sobrecarga laboral, siendo estos más proclives a exteriorizar la incidencia del acoso.

El presente estudio busca aportar en la precisión de esta compleja temática, que en su definición se confunde con otras formas de agresión, lo importante es definir los direccionamientos organizacionales que tienen implícito, actitudes percibidas como hostiles, el planteamiento inicial se realiza bajo un marco que define la situación actual en Colombia, planteando los objetivos y formulando la justificación y realización del estudio.

Para el estudio de las variables asociadas al acoso se realizaron encuestas de percepción de mobbing y autoevaluaciones para detectar el estilo de liderazgo característico del líder buscando establecer relaciones, los datos se presentan por equipos de trabajo con el fin de obtener información acerca de comportamientos que inciden en el acoso; finalmente se realiza un diagnóstico y se plantea acciones de mejora que pueden ser incorporadas dentro de las organizaciones. En última instancia, se presentan las conclusiones y las recomendaciones de acuerdo a los resultados globales, en concordancia con los objetivos planteados para el estudio.

\section{CONTENIDO}

\section{A. Generalidades del problema de investigación}

La organización contemporánea se ha enfrentado a escenarios competitivos, en que el trabajo coordinado entre esta y sus colaboradores es esencial para alcanzar el éxito proveniente entre dicha interacción que permite obtener resultados favorables en la productividad, por consiguiente, el presente y futuro de la organización depende sustancialmente de la 
adecuada administración del talento humano, factor dinámico de la misma.

Es fundamental que exista un clima de pacífica convivencia en el ambiente laboral, basado en la colaboración y el respeto; a través del buen trato, del reconocimiento de méritos, de la apropiada comprensión y la oportunidad de progreso, estos deben ser propósitos gerenciales visualizados como una inversión estratégica, factores claves para la consecución de los objetivos organizacionales, con el propósito de garantizar la sustentabilidad y sostenibilidad.

Durante décadas muchos autores han divulgado en sus escritos técnicas para dirigir y administrar el personal, es cierto que un buen desempeño en la organización, permite contribuir a la creación de ambientes de trabajo saludables donde son determinantes factores físicos organizacionales, y psicosociales, además de elementos culturales que inciden en 1 o s resultados productivos y que llevan a la organización hacia la excelencia y la competitividad.

Millones de trabajadores en el mundo se encuentran sometidos diariamente al acoso laboral, expuestos al "darwinismo social" que no apunta a la supervivencia del más competente sino del más despiadado, donde la enunciación meramente verbal de valores no es sustentable con acciones; hechos de terrorismo psicológico que han sido permitidos en Colombia y que forman fracturas en el tejido social e incitan a la competitividad individualista. Aunque el Acoso Laboral ya es un delito en Colombia, sucede solo 26 años después de que Suecia fuera pionero en incluir en su legislación el mobbing ${ }^{1^{1}}$ como una violación a la ley, la cual castiga el abuso físico y psicológico en el trabajo. En nuestro país se han decretado mediante la ley 1010 del $2006^{2^{2}}$ medidas para prevenir, corregir y sancionar el Acoso Laboral y otros hostigamientos en el marco de las relaciones de trabajo; en la que el Congreso de la Republica propone un mecanismo de regulación a un problema muy común y recurrente en las organizaciones, tratado con reserva y limitada apertura.

\footnotetext{
${ }^{1}$ Mobbing: Conducta no deseada que atente contra la dignidad de una persona y cree un entorno intimidatorio, humillante $\mathrm{u}$ ofensivo.

2 Colombia. Congreso de la republica. Ley 1010 de 2006: Diario Oficial No. 46.160, de 23 de enero de 2006. [en línea]. [consultado 5 ago. 2011]. Disponible en <http://www.secretariasenado.gov.co/senado/basedoc/ley/2006/ley_1010_2 006.html >
}

Desafortunadamente el común denominador de las culturas organizacionales restringe el uso del sistema normativo disponible en el país, con el cual los trabajadores evidencien el acoso laboral y sus efectos en la salud mental; limitaciones principalmente de tipo económicas como el temor a la pérdida del empleo por falta de oportunidades en el mercado laboral, impiden que este problema sea visible en la sociedad y que bajo técnicas de ataques sutiles el maltrato, la persecución, la discriminación, la inequidad y el entorpecimiento sean actos permitidos en las empresas. La no intervención efectiva ante episodios de violencia laboral, puede ocasionar consecuencias no deseadas en el trabajador como el estrés, la ansiedad, el aislamiento, baja autoestima, insomnio, incluso en algunos casos extremos el suicidio; trascendiendo esto a la organización mediante pérdidas económicas, desaprovechamiento de talentos, menor producción y hasta el desprestigio social, en síntesis un elemento destructivo del clima laboral y por consiguiente de su desarrollo organizacional.

\section{B. Metodología}

Tipo de Investigación

Investigación mixta de tipo cuantitativo y cualitativo no experimental, en cuanto no busca manipular deliberadamente las variables, es decir, no se intervienen las variables, sino que por el contrario se describe el fenómeno y como se da en su contexto natural, por esta razón se ha realizado bajo un diseño descriptivo de tipo correlacional con muestreo por conveniencia, lo cual ha permitido medir el grado de relación y la manera que interactúan entre sí las variables "Percepción de acoso laboral" y "Estilo de liderazgo". La presente permitirá identificar los retos metodológicos que involucra el estudio de estas relaciones, así como posibles conceptos o variables promisorias y prioridades para investigaciones posteriores.

En el planteamiento inicial se realizó una delimitación para la investigación en cuatro sectores (Textil, Metalmecánico, Alimentos y Servicios), cada uno asumido con el criterio de población y siendo abordadas inicialmente con la herramienta estadística de muestreo aleatorio simple. Este planteamiento inicial, pretendía a través del muestreo probabilístico asegurar la representatividad de la muestra elegida, este método otorgaba una probabilidad conocida de integrar la muestra a cada elemento de la población, lo que le aportaba confiabilidad al estudio. Para los marcos poblacionales se utilizaron las bases de datos suministradas por las Cámaras de Comercio de Pereira y Dosquebradas, en las cuales se eliminaron los registros de empresas que contaban menos de 15 colaboradores, dado que en las empresas pequeñas no se contaba con el número necesario de colaboradores para obtener mediciones de las variables de interés de este estudio. Por múltiples 
inconvenientes recurrentes, relacionados con la apertura de las empresas inicialmente definidas en la muestra y la negativa para la aplicación del instrumento, fue necesario modificar el criterio de muestreo propuesto en el diseño inicial, por un muestreo por conveniencia, aplicando los instrumentos a la mayor cantidad de organizaciones que permitieran la realización del estudio. Por la sensibilidad del tema las empresas seleccionadas en el muestreo inicial argumentaron no estar interesadas en participar en la investigación, sin embargo, surgieron las que permitieron el acceso y son éstas objeto de investigación.

\section{Variables e Indicadores}

\subsection{Dimensión: estilo de liderazgo.}

Teniendo en cuenta el concepto de que la investigación cualitativa pretende abordar la naturaleza profunda de las realidades en su sistema de relaciones y estructura dinámica; para la obtención de esta variable se ha optado por tomar un instrumento diseñado por Amauta Internacional $^{\mathbf{3}}$, el cual permite conocer las actitudes de aquellos líderes de equipos responsables de grupos estratégicos dentro de la organización. Es así como se realiza un diagnóstico con esta variable para proceder a relacionarla con la variable cuantitativa que se ha diseñado correspondiente a la percepción de Acoso Laboral.

La organización Amauta Internacional p r e s e n t a en uno de sus módulos llamado "Estilos de Facilitación y Motivación" las diversas formas de liderar mediante un modelo basado en etapas donde afirman que un estilo de facilitación o liderazgo "bueno" o "correcto" para un grupo en una etapa de desarrollo, no lo es para un grupo en otra etapa o para el mismo grupo cuando evoluciona o en su defecto involuciona. Dentro del modelo se sustenta que cada etapa requiere de diversas características de liderazgo debido a que las necesidades, las metas y los recursos del grupo son diferentes. Esta propuesta es muy acorde a la investigación propuesta, aunque existan textos que indiquen que solo hay una forma de liderar correctamente, este modelo basado en la experiencia de la organización expone cuatro, un estilo para cada etapa. En la aparte sistematización de variables se exploran cada uno de los estilos de liderazgo.

\subsection{Sistematización de variables}

\subsubsection{Dimensión: Percepción de acoso laboral}

3. Amauta Internacional. Formación de facilitadores para el desarrollo organizacional. Universidad Tecnológica de Pereira. Facultad de Ingeniería Industrial. 2005. Módulo 5 facilitación.
La investigación ha tenido además el propósito de diseñar y construir un instrumento para la medición de la Percepción del Acoso Laboral, riesgo psicosocial que ha tenido gran impacto últimamente en el medio Laboral.

\section{Variables asociadas al acoso Laboral:}

Entorpecimiento Laboral: Corresponde a la obstaculización de las funciones y responsabilidades para el cumplimiento de la labor o hacerla más gravosa o retardarla con perjuicio para el colaborador.

Persecución Laboral: Todo acto de violencia contra la integridad física o moral, la libertad física o sexual y los bienes de quien se desempeñe como empleado o trabajador; toda expresión verbal injuriosa o ultrajante que lesione la integridad moral o los derechos a la intimidad y al buen nombre de quienes participen en una relación de trabajo de tipo laboral o todo comportamiento tendiente a menoscabar la autoestima y la dignidad de quien participe en una relación de trabajo de tipo laboral.

Marginación Laboral: Toda conducta cuyas características de reiteración o evidente arbitrariedad permitan inferir el propósito de inducir la renuncia del empleado o trabajador, mediante la descalificación, la carga excesiva de trabajo y cambios permanentes de horario que puedan producir desmotivación laboral.

Inequidad Laboral: Favoritismo que carezca de razonabilidad desde el punto de vista laboral, asignación de funciones a menosprecio del trabajador. Inequidad es la falta de equidad o de igualdad. Cuando no son equitativos ósea justos e igualitarios, es la carencia de igualdad en el ámbito social.

Burnout: De s g a s te profesional derivado de malas prácticas gerenciales, consiste en el resultado de un proceso de estrés crónico laboral y organizacional que termina en un estado de agotamiento emocional y de fatiga desmotivante para las tareas laborales.

\section{Variables asociadas a los estilos de liderazgo:}

Estilo Directivo: (Etapa Reactiva): En esta etapa, donde prima la necesidad sentida de sobrevivir a una amenaza a la existencia misma del sistema y de la vida de sus integrantes, la facilitación más efectiva es la de un liderazgo protagónico. El facilitador de esta etapa logra su mayor efectividad con un estilo directivo y aun autocrático dentro de unas estructuras y reglas claras, realistas y obligatorias, siempre que éstas sean justas. Lo anterior no quiere decir que el facilitador deba ser déspota; al contrario, debe ser motivado por una sincera compasión y empatía por el grupo y una sensibilidad a su sufrimiento. El líder-facilitador continuamente resalta, entre el grupo, el valor 
de la vida y de haber sobrevivido la amenaza, como punto de partida para el restablecimiento de la autoestima individual y grupal.

Estilo Coordinador: (Etapa Receptiva) Superada la crisis inmediata, el sistema dedica su energía a la creación de una infraestructura mediante la cual sus integrantes pueden satisfacer sus necesidades básicas de seguridad. La filosofía de intervención ya no se debe centrar en conductas paternalistas o asistencialistas, sino en facilitarles la adquisición de los conocimientos y las destrezas necesarias para que ellos hagan por sí mismos lo que necesita hacer para satisfacer sus necesidades básicas. El líder efectivo a este nivel es el que facilita una relación interpersonal que va más allá de la tolerancia. Se requiere de una relación basada en el respeto mutuo, con el fin de lograr un proceso de concertación que logre una aceptación explícita y no sólo táctica. Se requiere que cada participante en el grupo, o al menos la mayoría de ellos, acepte abierta y claramente la "oferta" de liderazgo y/o de ayuda, y se comprometa a cumplir las condiciones y exigencias del líder, también aceptando las consecuencias de no hacerlo.

Estilo Monitor: (Etapa Proactiva) Cuando un grupo social o comunitario comienza a establecer sus bases de seguridad durante la segunda etapa, comienza a nacer en él la necesidad de autonomía, la independencia como grupo, posiblemente antes de que el mismo haya adquirido los conocimientos y las destrezas necesarias para desarrollarla adecuadamente. En esta etapa, el líder se compromete con los integrantes a facilitar que ellos "sean y crezcan", mediante procesos autogestionarios debidamente dosificados en la medida en que el grupo esté preparado para asumir cada nueva responsabilidad. La meta es poder dejar atrás todo vestigio de autoritarismo para que todos se rijan por un sistema democrático en el que las estructuras y reglas son concertadas, aceptadas y modificadas entre todos. La retroalimentación del facilitador también debe tomar un viraje radical. En lugar de la evaluación y el juicio de la segunda etapa, el grupo que está desarrollando esta etapa requiere una retroalimentación progresivamente más descriptiva y relativista.

Estilo Asesor: (Etapa Interactiva): No son muchos los grupos que logran desarrollar una masa crítica en esta etapa porque es en la que hay que extender el proceso sinérgico interno a otros grupos y a los contextos sociopolíticos. El facilitador es sólo el Asesor de un grupo auto-dirigido, cuyo objetivo es el de lograr una integración macro-sistémica y crear redes sociopolíticas efectivas para lograr una verdadera co-evolución, subcultural, cultural y, de hecho, Mediante sus estructuras matriciales, la corrección es una total auto-corrección y en una constante y mutua retroalimentación. Es la responsabilidad de todos proteger la colectividad, sin dejar de aportar y defender los espacios de cada individuo.

\subsection{Instrumentos de recolección de información ${ }^{4}$}

Autoevalaución estilos de liderazgo: Instrumento tomado de Amauta Internacional que categoriza al líder entre cuatro posibles estilos: directivo, coordinador, monitor y asesor; siendo predominante el que obtenga la mayor puntuación. De igual manera la herramienta permite determinar condiciones de flexibilidad para transitar de un estilo a otro, este valor numérico se calcula con el mismo instrumento y se califica de acuerdo a los siguientes criterios.

Percepción de Acoso Laboral: El Instrumento está conformado por 44 ítems cada uno con cinco alternativas de respuesta que describen la percepción de actos hostiles que pueden o no presentarse en la organización; la percepción del Mobbing se evalúa a través de cinco variables, que para efectos de la descripción de los resultados se estableció una escala likert, herramienta que facilita la cuantificación de una variable cualitativa.

Las variables definidas para fueron operacionalizadas así:

\begin{tabular}{|c|c|}
\hline VARIABLE & INDICADOR \\
\hline \multirow{6}{*}{$\begin{array}{l}\text { Entorpecimiento } \\
\text { laboral }\end{array}$} & Obstaculización a solución de problemas \\
\hline & Modificación arbitraria de las funciones del cargo \\
\hline & $\begin{array}{lllll}\begin{array}{l}\text { Intimidación } \\
\text { normativos }\end{array} & \text { con } & \text { aspectos } & \text { disciplinarios } & \text { y } \\
\end{array}$ \\
\hline & Inequidad en la distribución de las actividades \\
\hline & $\begin{array}{l}\text { Ausencia de reconocimiento frente a logros } \\
\text { particulares del rol. }\end{array}$ \\
\hline & Evaluaciones de desempeño sesgadas \\
\hline \multirow{6}{*}{$\begin{array}{l}\text { Persecución } \\
\text { Laboral }\end{array}$} & Comunicación verbal y no verbal agresiva \\
\hline & Acceso limitado al líder \\
\hline & Llamados de atención injustificados \\
\hline & Extralimitación en el uso de la normatividad \\
\hline & Presión \\
\hline & Acoso sexual \\
\hline \multirow{3}{*}{$\begin{array}{l}\text { Marginación } \\
\text { Laboral }\end{array}$} & Invalidación de opiniones e iniciativas \\
\hline & Trasladar responsabilidades propias al colaborador \\
\hline & Decisiones tendenciosas que afectan el desempeño \\
\hline \multirow{3}{*}{$\begin{array}{l}\text { Inequidad } \\
\text { Laboral }\end{array}$} & Exclusión de proyectos relacionados con el cargo \\
\hline & Preferencias \\
\hline & Discriminación \\
\hline \multirow{2}{*}{ Burnout } & Perjuicio laboral \\
\hline & Estrés laboral \\
\hline
\end{tabular}

Tabla 1.Operacionalización de Variables Acoso Laboral

Fuente Las autoras

4 Amauta Internacional. Módulo 5. Cuestionario de autoevaluación estilos de liderazgo. Universidad Tecnológica de Pereira. 2005 


\section{RESULTADOS}

Los datos fueron suministrados por 112 colaboradores de empresas privadas de las ciudades de Pereira y Dosquebradas, para medir la percepción en los niveles tácticos de las mismas en cuanto al acoso laboral, al igual participaron 22 líderes de cada uno de los equipos de trabajo encuestados para obtener resultados acerca de los estilos de liderazgo predominantes y realizar la correlación entre las variables de interés.

Para efectos del análisis de los resultados obtenidos a través de la aplicación del instrumento "Percepción de Acoso Laboral" se hace la presentación de las respectivas tablas que registran frecuencias absolutas expresadas en calificaciones que van de 1 a 5 , y relativas que muestran la concentración de los puntajes evidenciando la masa crítica de la percepción y comportamiento de cada variable y sus indicadores.

Con el propósito de categorizar los puntajes se han establecido rangos que determinan la ubicación de la percepción de acoso de la siguiente manera: Puntajes entre 1 y 2 no se manifiestan percepciones asociadas al acoso, puntajes entre 2 y 3 alerta media-baja, puntajes entre 3 y 4 alerta media-alta y puntajes entre 4 y 5 presencia de percepciones de acoso.

\begin{tabular}{|c|c|}
\hline $1-2$ & No existe comportamiento de acoso \\
\hline $2-3$ & Alerta Media -Baja \\
\hline $3-4$ & Alerta Media -Alta \\
\hline $4-5$ & Comportamiento de acoso \\
\hline
\end{tabular}

Tabla 2. Categorización de puntajes percepción de acoso Fuente Las autoras.

\section{Resultados Acoso Laboral (Mobbing):}

El instrumento de percepción da la posibilidad de realizar un registro de datos para interpretar la presencia de mobbing como condición que conjuga los diversos comportamientos de acoso laboral; Una vez realizado el trabajo de campo se encuentra que en general no existe una percepción de acoso en la muestra objeto de investigación, sin embargo es importante evidenciar que si bien no son resultados contundentes si se aprecian alertas medio bajas y alertas bajas que denotan vulnerabilidad y riesgos que de no ser atendidos pueden generar comportamientos de acoso a futuro, desde los referentes teóricos de sustenta que eventos asociados al acoso son calificados como riesgos psicosociales que pueden a mediano y largo plazo patologías de orden psicológico y físico. Se encuentran como variables generales configuradas como alertas medio bajas y baja el entorpecimiento laboral, la inequidad y la marginación laboral, sutiles en comparación con la persecución y el burnout que pueden llegar a ser más evidentes por sus graves y visibles consecuencias Psicosociales.
En la siguiente gráfica el comportamiento global del fenómeno en las organizaciones analizadas.

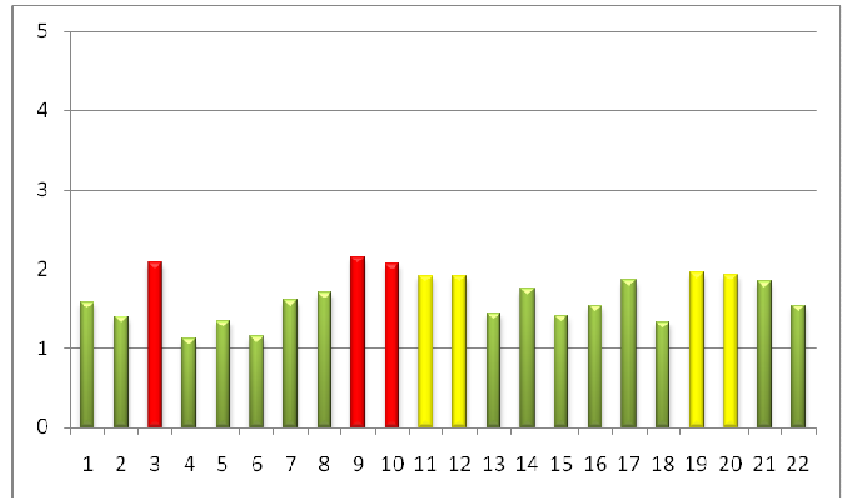

Figura 1. Gráfico de barras resultados percepción de acoso en la muestra

Fuente las autoras

\begin{tabular}{|l|l|}
\hline & Alerta media baja de acoso laboral \\
\hline & Alerta baja de acoso laboral \\
\hline & No existe comportamiento de Acoso laboral \\
\hline
\end{tabular}

Tabla 3. Criterios de interpretación resultados finales percepción de acoso

Fuente Las autoras.

\section{Resultados estilos de liderazgo:}

Con el siguiente gráfico se muestran los estilos de liderazgo registrados por los 22 líderes participantes en la investigación, evidenciando en cada barra por segmentos el porcentaje de cada uno de los estilos, haciendo visible el estilo predominante para cada uno, Como segundo análisis se realiza una comparación entre el comportamiento de los resultados graficados en cada barra reflejando la concentración de estilos entre todos los líderes. Se puede determinar entonces, la presencia del estilo coordinador como común denominador entre los líderes evaluados, lo que implica que los equipos están en fase de entrenamiento y requieren una presencia constante de acompañamiento, no obstante, en el análisis individual se registra el uso de este estilo en equipos que no lo requieren, lo que puede predisponer y facilitar percepciones de entorpecimiento laboral y poca libertad para el ejercicio laboral. Por otro lado, en el comportamiento de los datos se observa un resultado del estilo directivo que sin ser el más predominante ocupa la siguiente franja de concentración en el resultado, lo que propone una presencia protagónica del líder donde se restringen las posibilidades de actuación en un marco de autonomía y autorregulación, el uso de este estilo es propicio para momentos de crisis y de surgimiento de un sistema; situación contraria a la mayoría de las organizaciones analizadas. Se observa una limitada presencia con relación a los estilos de liderazgo monitor y asesor, donde la actuación de los lideres debe ser menos protagónica en un sistema facultado para actuar con autonomía, empoderamiento e independencia. 
Finalmente se puede inferir que, si bien no existe una correlación directa entre estilo de liderazgo y percepción de acoso, es importante que cada uno de los lideres identifique plenamente el momento de evolución de los equipos y de esta manera establecer el estilo de liderazgo propicio. Para hacer más claro el resultado se presenta a continuación el histograma de frecuencias como gráfico apilado para comparar las categorías "estilos de liderazgo":

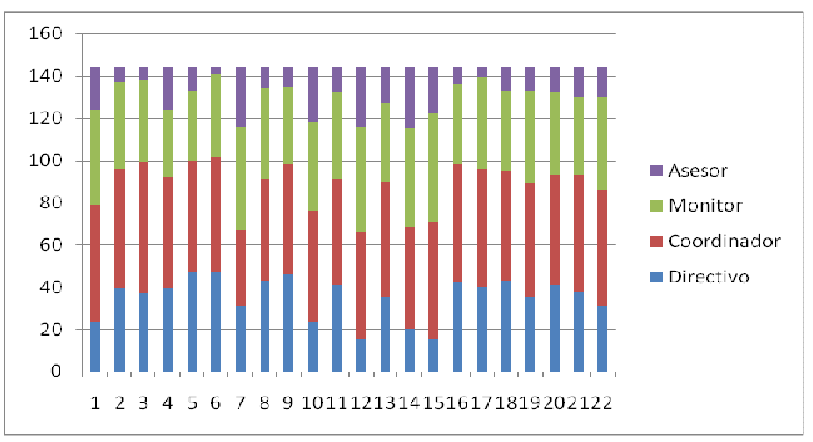

Figura 2. Estilos de liderazgo líderes de cada uno de los grupos Fuente las autoras.

\section{CONCLUSIONES}

Aunque el tema de acoso laboral, se encuentra normalizado y regulado a través de la Legislación Laboral Colombiana, y siendo un tema de cumplimiento obligatorio en lo que respecta a la Salud Ocupacional y en particular al riesgo psicosocial, aún se encuentra una gran resistencia en las organizaciones, lo que evidencia ausencia de información y probablemente temor para enfrentar realidades culturales en la empresa, que involucren la competencia del líder y sus efectos en el desarrollo del talento humano. La resistencia en las organizaciones para identificar comportamientos acosadores da cuenta de ello.

Respecto a las características del instrumento diseñado y aplicado para la medición de percepción de mobbing, los resultados sustentan que es viable para evaluar los diferentes aspectos del acoso laboral en función de los criterios existentes en la legislación Colombiana; y de las conductas específicas encontradas en la literatura, en síntesis el mobbing es susceptible de medición.

El estilo facilitador predominante en los 22 líderes que participaron en la investigación es el coordinador, lo cual permite suponer que de los estándares educativos a los que están sujetos los gerentes organizacionales de hoy, forman estilos de liderazgo que siendo efectivos en su actuación pueden generar grupos de trabajo dependientes, que a la luz de los enfoques administrativos actuales son poco apropiados, para ejercer un rol de liderazgo proactivo se deben entender las diferencias individuales, culturales y generacionales, tener claro que se lideran personas, no procesos, al igual que tener presente que en los direccionamientos organizacionales la meta es crear líderes, no seguidores, siendo la formación académica y profesional clave en esta misión.

Constantemente se escuchan expresiones acerca de lo desagradable e injusta que puede resultar una actividad laboral, el desarrollo de la investigación expone conductas que demuestran la existencia de aspectos que van más allá de los conflictos comunes en el ámbito laboral, y que, por tanto, ameritan ser identificadas, tipificadas y corregidas.

Cuando el conflicto ha sido detectado y adquiere un carácter disfuncional que afecta la normalidad de los procesos y sus resultados, se pueden sustituir las metas y recompensas que resulten "competitivas" por otras que demanden cooperación entre los colaboradores, con el propósito de eliminar situaciones que sean detonantes de confrontaciones y deterioren el clima organizacional y el sano ambiente laboral.

No se puede afirmar desde los resultados que tanto el estilo de liderazgo directivo como el coordinador son generadores de actos hostiles, se corrobora la teoría en cuanto a que la utilización de estos es adecuada dependiendo del momento de evolución de los equipos, siempre y cuando estén enmarcados en los lineamientos conductuales y espaciales del grupo de trabajo; es decir, si el desarrollo del liderazgo no corresponde al momento de evolución del equipo su utilización puede ser susceptible a la percepción de acoso.

El compromiso de la alta dirección tanto en la fase de diagnóstico como en el tratamiento fortalece las relaciones interpersonales y brinda un ambiente de confianza en la organización, dando apertura a los colaboradores en el planteamiento de inquietudes que serán tratadas con disposición.

\section{RECOMENDACIONES}

Sensibilizar a los futuros líderes desde la academia en su proceso formativo acerca de la importancia de interpretar, e identificar situaciones relacionadas con el acoso, lo que permita en posteriores ejercicios de investigación más apertura por parte de las empresas para la identificación de realidades que pueden ser tratadas mediante intervenciones que contribuyan a la generación de climas organizacionales sanos y estudios científicos con más precisión acerca del tema.

Sensibilizar acerca de la importancia de conocer la legislación colombiana en términos laborales, y en particular lo que respecta a los riesgos psicosociales, para obtener herramientas que permitan a los trabajadores salvaguardar su integridad conociendo los límites existentes en el trato líder- colaborador.

Evitar considerar los conflictos como algo que debe eludirse, 
por el contrario, interpretarlos como un fenómeno normal e inevitable que construye oportunidades de mejora, siempre y cuando sean tratados de manera adecuada, para lo que se requiere formación de los lideres organizacionales.

Conocer las herramientas legales existentes en Colombia, hacer uso de ellas, exigiendo los derechos que se tienen dentro del ámbito laboral, de esta manera pueden limitase la actuación de los acosadores, que no se generan solo en la dirección líder colaborador, también puede presentarse desde el colaborador hacia en líder y entre colegas y compañeros de equipos de trabajo.

Contar con métodos para detectar, diagnosticar y tratar los conflictos que surgen en el día a día organizacional, deteniendo de esta forma la aparición de actos hostiles que lleven a la práctica del mobbing, es importante y necesario, permitiendo a las organizaciones contar con ambientes psicosociales sanos y seguros.

Complementar el presente estudio desde la perspectiva del acosado, obteniendo información por parte de entidades de salud o instancias jurídicas, de las cuales se pueda tener de primera mano información relacionada directamente al acoso, para inferir en las implicaciones de este acto en la vida laboral y los efectos que puede causar en la productividad y rentabilidad de la organización.

\section{REFERENCIAS}

$\underline{\text { Referencias de libros: }}$

[1] CHIAVENATO, Idalberto. Introducción a la teoría general de la administración. 7 ed. México: McGraw - Hill, 2007, p.562

[2] Gestión del talento humano. 3 ed. México: McGraw - Hill, 2009, p. 475

[3] HELLRIEGEL, Don y SLOCUM, John W. Comportamiento organizacional. 10 ed. México: International Thompson, 2004, p.524

[4] Comportamiento organizacional. $12 \mathrm{ed}$. México: Cengage Learning, 2009, p 640

[5] LEYMAN, Heinz. Mobbing. La persécution au travail. Citado por PIÑUEL, Iñaki. Mobbing: cómo sobrevivir al acoso psicológico en el trabajo. 1 ed. España: Sal Terrae, 2001, p. 312

[6] LUSSIER, Robert y ACHUA Christopher. Liderazgo. 2 ed. México: International Thomson, 2005, p. 498

Documentos presentados en conferencias (No publicadas aún):
[7] COLOMBIA. CONGRESO DE LA REPUBLICA. Ley 1010 de 2006: Diario Oficial No. 46.160, de 23 de enero de 2006. [en línea]. [consultado 5 ago. 2011]. Disponible en <http://www.secretariasenado.gov.co/senado/basedoc/ley/2 006/ley_1010_2006.html>

[8] JIMÉNEZ MORALES, Germán. Infiernos Laborales. En : El Colombiano, Medellín: (11, abr., 2010). [en línea]. (1998). [consultado 19 Oct. 2011]. Disponible en <http://www.elcolombiano.com/BancoConocimiento/I/infie rnos_laborales/infiernos_laborales.asp >

[9] WATKINS, Dave. An Application Framework for Talent Management That Acts as a Central Feedback Center for all Organizational Functions. En: IT Tool. [en línea]. (1998). [consultado 12 sep.2011]. Disponible en <http://www.hreonline.com/pdfs/03012008SoftscapeDocu ment.pdf $>$

[10] WIL HARZING, Anne. On becoming a high impact journal in international business and management. En : European Journal of International Management. [En línea]. Vol. 2, No. 2, (2008). [consultado23 ago.2011]. Disponible en $\quad$ http://www.h arzing.com/download/impactejim.pdf> 